\title{
Clustering Approach for Colour Image Enhancement using Stationary Wavelet Transformation
}

\author{
K. Baranitharan \\ Research Scholar \\ St Peter's University \\ Chennai, India
}

\author{
S. Srinivasa Rao Madane, Ph.D \\ Principal \\ Adhiparasakthi College of Engineering \\ Kalavai, Vellore, India
}

\begin{abstract}
Image enhancement is a technique that enhances the perceptual quality of the images and restraining other information on it. The images with enhanced perceptual quality are needed in various areas of application such as remote sensing, medical and aerial image analysis. In this study a robust colour image enhancement technique is proposed by exploiting Stationary Wavelet Transform (SWT), HSV (Hue, Saturation and Value) colour model, $k$-means clustering, hill climbing and Histogram Equalization (HE) techniques. A visible improvement in the image quality for human contrast perception is achieved by this proposed approach and the objective assessment also provides satisfactory results.
\end{abstract}

\section{General Terms}

Clustering, Enhancement, Signal Decomposition.

\section{Keywords}

Stationary Wavelet Transformation, Hill climbing algorithm, Clustering approach, HSV colour model.

\section{INTRODUCTION}

Image enhancement technique improves the information content of the images and sharpens the image features to obtain better visual quality. In this section, existing colour image enhancement approaches are discussed.

Space variant luminance map (SVLM) based colour image enhancement technique is presented in [1]. The RGB image is converted into intensity image. Then the intensity image is low pass filtered using a 2D discrete Gaussian filter to estimate its luminance.2D gamma correction combined with the SVLM is applied for luminance enhancement. The enhanced luminance information is applied to adaptive contrast enhancing process and the linear colour restoration. An image enhancement technique for colour images is described in [2]. To achieve enhancement, RGB colour space is converted into HIS colour space initially. After transformation, the hue component is kept unchanged and saturation component is stretched exponentially. The intensity component is enhanced using pulse couple neural network. Finally, HIS to RGB conversion is taken place using the updated components.

A new scheme for colour image enhancement based on directional transform using wavelet basis and Gaussian low pass filters is presented in [3]. To enhance the given image, wavelet decomposition is applied to obtain flat and edge regions and then adaptive shrinkage is applied. Adaptive morphological filter based colour image enhancement is implemented in [4]. New type of opening and closing operators are designed and it can alter its shape according to the local geometry of the processed images.Virtual histogram approach based colour image enhancement is presented in [5].The given RGB image is transformed into $\mathrm{YC}_{b} \mathrm{C}_{\mathrm{r}}$ colour space, where local and global processing takes place on luminance and chrominance components of the image. A new adaptive contrast enhancement is developed in [6] for image enhancement based on Local Standard Deviation (LSD). First mathematical Hunt's image model is used for LSD distribution. Then, the contrast gains (CG) are formulated as a function of the LSD and an adequate CG is obtained by non linear LSD function.

Brightness preserving dynamic histogram equalization based colour image enhancement technique is described in [7]. The RGB colour space is converted into HSI and only the intensity channel is processed. Fuzzy intensification based colour image enhancement is discussed in [8]. Image information is modelled in spatial domain by using Gaussian membership function. New contrast intensification operator $t$ is involved for colour image enhancement. The parameter $t$ is calculated globally, by minimizing the fuzzy entropy of the image information.

A new method for colour image enhancement technique in Discrete Cosine Transform (DCT) domain is discussed in [9]. The enhancement technique is performed by three steps; adjustment of background information, preservation of local contrast and preservation of colour. All the steps are performed in a block DCT space. A new way of image enhancement approach is implemented using transform coefficient histogram [10]. Three methods of image enhancement algorithms; logarithmic transform histogram matching, logarithmic transform histogram shifting and logarithmic transform histogram shaping using Gaussian distributions are described based on logarithmic transform domain histogram and HE.

An automatic and parameter free contrast enhancement algorithm for colour images is presented based on piecewise linear transformation [11]. The luminance component is investigated by image content analysis algorithm to obtain the image illumination distributions. The parameters computed from the distributions are used in piecewise linear transformation function to enhance the images. An advanced image contrast enhancement approach is discussed in [12] based on non overlapped sub-block HE function. It is obtained by employing a low pass filter type mask. It produces the high contrast associated with local HE.

Recursive rational filter based colour image enhancement is presented in [13]. To control the dynamics effects in image, suitable nonlinear function is applied to the illumination component and the details can be enhanced by processing the reflectance. 
In this study, an approach for colour image enhancement based on SWT is presented. The rest of the paper is structured as follows: The groundwork of the proposed system is based on SWT and k-means clustering. The brief descriptions of the proposed system are explained in section 2. Experimental results and conclusions are discussed in section 3 and 4 respectively.

\section{METHODS AND MATERIALS}

The main goal of the proposed colour image enhancement system is to improve the visibility of image details without producing any unrealistic visual perception or artifacts by using SWT analysis. The proposed colour image enhancement system composed of three computational blocks: Colour space conversion, SWT decomposition and enhancement block. The overall computational block of the proposed colour image enhancement system is shown in Figure 1.

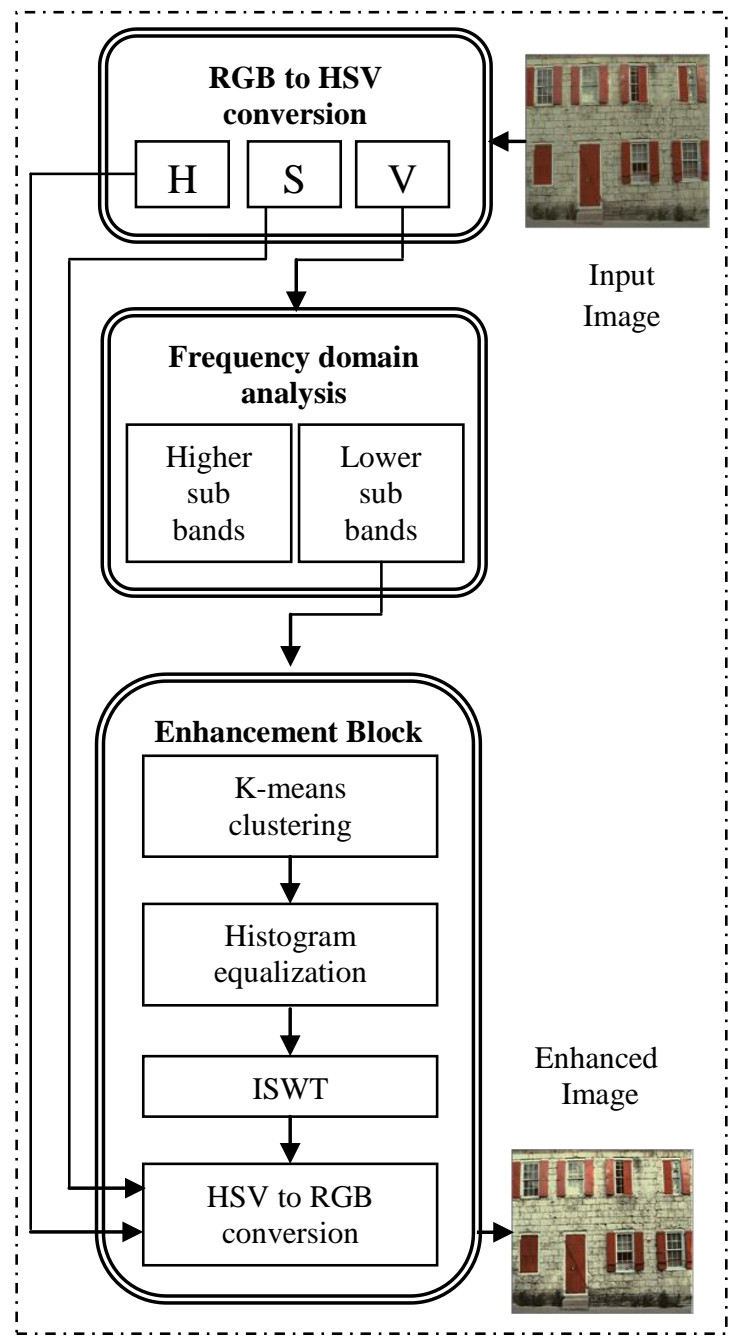

Fig1: Block diagram of the proposed colour image enhancement system

Initially, the given RGB colour image is transformed into HSV colour model in the colour space conversion block. HSV space is very closer to human perception in which the $\mathrm{H}$ refers the spectral composition of colour, $\mathrm{S}$ indicates the purity of colours and $\mathrm{V}$ defines the brightness of a colour or just the luminance value of the colour. The transformation of RGB to HSV colour model is as follows [14]:

$$
\begin{gathered}
\mathrm{S}=\frac{\max (\mathrm{R}, \mathrm{G}, \mathrm{B})-\min (\mathrm{R}, \mathrm{G}, \mathrm{B})}{\max (\mathrm{R}, \mathrm{G}, \mathrm{B})} \\
\mathrm{V}=\max (\mathrm{R}, \mathrm{G}, \mathrm{B})
\end{gathered}
$$

if $R=\max (R, G, B)$ and $G=\min (R, G, B)$

$$
\mathrm{H}=5+\mathrm{B}^{\prime}
$$

else if $R=\max (R, G, B)$ and $G \neq \min (R, G, B)$

$$
\mathrm{H}=1-\mathrm{G}^{\prime}
$$

else if $G=\max (R, G, B)$ and $B=\min (R, G, B)$

$$
\mathrm{H}=\mathrm{R}^{\prime}+1
$$

else if $\mathrm{G}=\max (R, G, B)$ and $B \neq \min (R, G, B)$

$$
\mathrm{H}=3-\mathrm{B}^{\prime}
$$

else if $R=\max (R, G, B)$

$$
\mathrm{H}=3+\mathrm{G}
$$

otherwise

$$
\mathrm{H}=5-\mathrm{R}^{\prime}
$$

$\mathrm{H}$ is then converted to degrees by multiplying by 60 giving HSV with $\mathrm{S}$ and $\mathrm{V}$ between 0 and 1 and $\mathrm{H}$ between 0 and 360 .

$$
\begin{aligned}
& \text { Whereas } \mathrm{R}^{\prime}=\frac{\max (\mathrm{R}, \mathrm{G}, \mathrm{B})-\mathrm{R}}{\max (\mathrm{R}, \mathrm{G}, \mathrm{B})-\min (\mathrm{R}, \mathrm{G}, \mathrm{B})}, \\
& \mathrm{G}^{\prime}=\frac{\max (\mathrm{R}, \mathrm{G}, \mathrm{B})-\mathrm{G}}{\max (\mathrm{R}, \mathrm{G}, \mathrm{B})-\min (\mathrm{R}, \mathrm{G}, \mathrm{B})} \text { and } \\
& \mathrm{B}^{\prime}=\frac{\max (\mathrm{R}, \mathrm{G}, \mathrm{B})-\mathrm{B}}{\max (\mathrm{R}, \mathrm{G}, \mathrm{B})-\min (\mathrm{R}, \mathrm{G}, \mathrm{B})}
\end{aligned}
$$

Subsequent to HSV colour space conversion, the ' $\mathrm{V}$ ' channel is considered for frequency domain analysis as it contains the luminance information. SWT is adapted as frequency domain transformation, which is used to overcome the lack of translation invariant property of wavelet transform. In addition to this advantage, it has no decimation property [15]. The procedure for decomposing an image by undecimated wavelet transform is same as discrete wavelet transform. However, the main difference in undecimated wavelet transform is, it omits both down-sampling in the forward and up sampling in the inverse wavelet transform. It produces approximation and detailed sub bands. As the approximation sub-band is the approximate of the original input image, it is only taken into account for clustering process in the enhancement process

In order to achieve better clustering outcome, $k$-means segmentation is employed, whereas hill climbing approach is adapted for automated peak $(k)$ prediction in enhancement block. To facilitate the enhanced colour image, HE is applied independently on each clustered image. Then, enhanced low frequency clustered sub band undergoes into inverse SWT along with high frequency sub bands to bring back the input image in spatial domain .Eventually, the enhanced colour image is achieved in RGB colour space by transforming back from HSV to RGB colour model. 

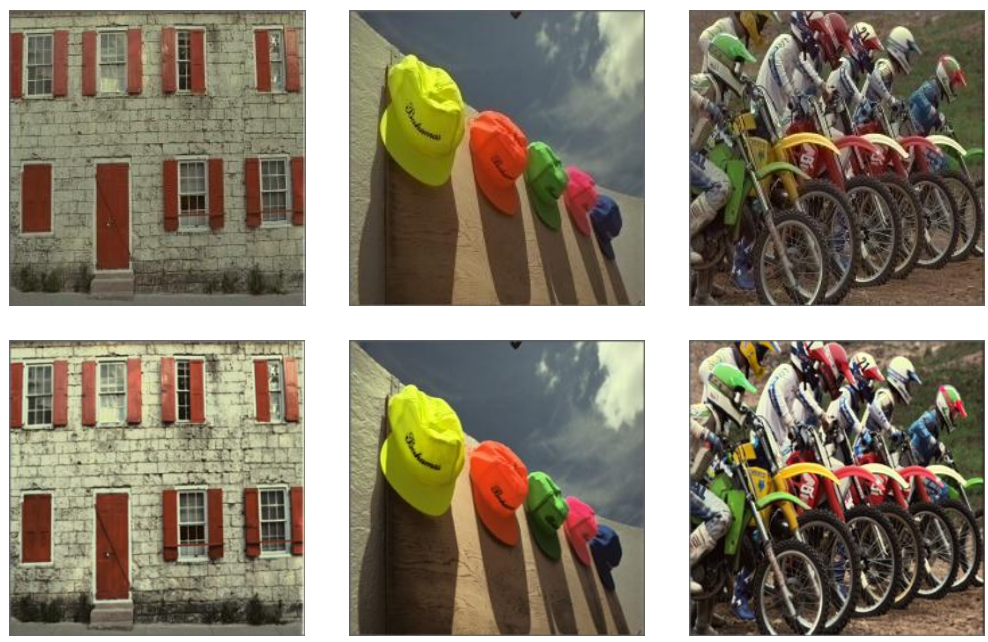
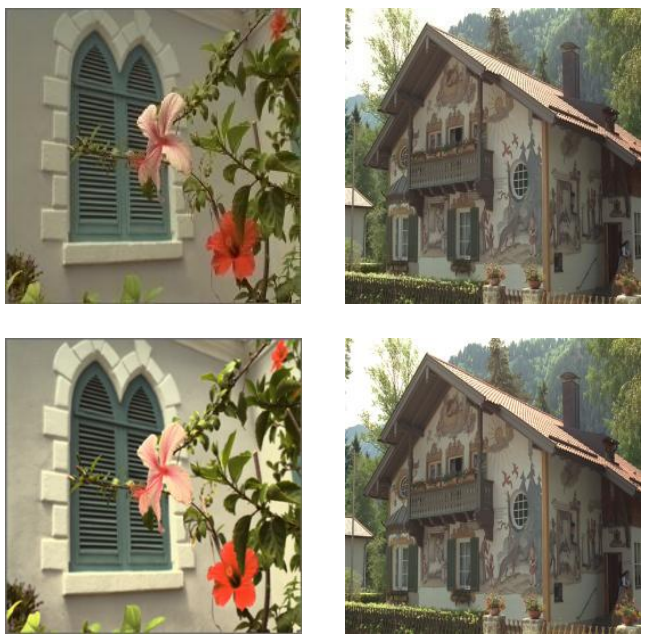

Fig 2: First row: Original images, Last row: Enhanced images by the proposed approach

\section{EXPERIMANTAL RESULTS}

In order to assess the performance of the proposed colour image enhancement system, MICT database images [16] are taken into account. Figure 2 shows the enhancement results of the proposed system for test images in MICT database images. Also, the generally used four quality parameters such as Peak Signal to Noise Ratio (PSNR), Structural SIMilarity (SSIM) index, Universal Quality Index (UQI), and Entropy are computed for performance evaluation. It is clearly observed from the figure that the proposed system provides better visual perception with superior colour conservation. Also it preserves finer details of the images without any distortions. In addition to the visual perception analysis, objective assessment is also considered by computing some statistical parameters such as PSNR, SSIM, UQI and Entropy. The obtained performance metrics of all images in MICT database is shown in Table 1 .

Table 1. Results of the proposed method for MICT database

\begin{tabular}{|c|c|c|c|c|}
\hline Images & PSNR & SSIM & UQI & ENTROPY \\
\hline 1 & 40.1543 & 0.9075 & 0.9508 & 7.6106 \\
\hline 2 & 38.0691 & 0.9567 & 0.9659 & 7.4041 \\
\hline 3 & 35.5018 & 0.9000 & 0.9070 & 7.6694 \\
\hline 4 & 35.7068 & 0.9638 & 0.9760 & 7.5579 \\
\hline 5 & 48.8044 & 0.8985 & 0.9496 & 7.7307 \\
\hline 6 & 33.8872 & 0.9657 & 0.9595 & 7.8377 \\
\hline 7 & 41.6645 & 0.9886 & 0.9989 & 7.2012 \\
\hline 8 & 34.8962 & 0.9224 & 0.9461 & 7.7291 \\
\hline 9 & 50.1663 & 0.9657 & 0.9959 & 7.3845 \\
\hline 10 & 39.5276 & 0.9790 & 0.9883 & 5.9050 \\
\hline 11 & 35.2713 & 0.9487 & 0.9927 & 7.2302 \\
\hline 12 & 38.1508 & 0.9557 & 0.9817 & 7.4355 \\
\hline 13 & 42.7908 & 0.9912 & 0.9956 & 7.4530 \\
\hline 14 & 33.1780 & 0.9284 & 0.9508 & 7.5644 \\
\hline Avg & 39.1264 & 0.9480 & 0.9685 & 7.4081 \\
\hline
\end{tabular}

It is evident from the Table 1 that the proposed approach achieves better SSIM (close to 1) and PSNR values. The maximum value of SSIM and PSNR indicates the high similarity and better reconstruction ability between the given image and enhanced image. Also, the other image quality parameters like UQI and entropy also yields better results.

\section{CONCLUSION}

A robust enhancement scheme is proposed in this study based on SWT approach for visual quality enrichment of digital colour images. On account of image enhancement the given RGB image is initially transformed into HSV colour space. In order to avoid colour distortion, value or luminance channel is alone subjected into SWT decomposition wherein lower band coefficients only considered for further process. The lower band coefficients are clustered by $k$-means clustering technique, where hill climbing approach is utilized for automated peak selection. Consequently, each clustered partition is enhanced by HE approach. Then, the enhanced value component is converted back to RGB along with hue and saturation components following to inverse SWT. Experimental results show that the proposed system enhances the given image with very good visual quality and achieves over 94\% SSIM and UQI with very good PSNR. In future, discrete shearlet transform and discrete curvelet transform may be employed in order to improve the image quality.

\section{REFERENCES}

[1] Lee, S., Kwon, H., Han, H., Lee, G., and Kang, B, “A space-variant luminance map based color image enhancement", IEEE Transactions on Consumer Electronics, 2010, 56(4), pp.2636-2643.

[2] Zhang, Y., Wu, L., Wang, S., and Wei, G, "Colour image enhancement based on HVS and PCNN", Science China Information Sciences, 2010, Vol.53, No.10, pp.19631976.

[3] Kim, S., Kang, W., Lee, E., and Paik, J, "Waveletdomain color image enhancement using filtered directional bases and frequency-adaptive shrinkage", IEEE Transactions on Consumer Electronics, 2010, Vol.56, No.2, pp.1063-1070.

[4] Deng, W.P., Cheng, F., and Venetsanopoulos, A. N, "Adaptive morphological filters for colour image enhancement", Journal of Intelligent and Robotic Systems, 1996, Vol.15, No.2, pp.181-207.

[5] Xu, Z., Wu, H. R., Yu, X., and Qiu, B., "Colour image enhancement by virtual histogram approach", IEEE Transactions on Consumer Electronics, 2010, Vol.56, No.2, pp. 704-712.

[6] Chang, Dah, C., Wen, R., and Wu, "Image contrast enhancement based on a histogram transformation of 
local standard deviation", IEEE Transactions on Medical Imaging, 1998, Vol.17, No.4, pp.518-531.

[7] Kong, N. S. P., and Ibrahim, H., "Color image enhancement using brightness preserving dynamic histogram equalization", IEEE Transactions on Consumer Electronics, 2008, Vol.54,No.4,pp. 19621968.

[8] Hanmandlu, M., Jha, D., and Sharma, R., "Colour image enhancement by fuzzy intensification", Pattern recognition letters, 2003, Vol. 24, No.1, pp.81-87.

[9] Mukherjee, J., and Mitra, S. K., "Enhancement of color images by scaling the DCT coefficients", 2008, IEEE Transactions on Image Processing, Vol.17, No.10, pp.1783-1794.

[10] Agaian, S. S., Silver, B., and Panetta, K. A, "Transform coefficient histogram based image enhancement algorithms using contrast entropy", IEEE Transactions on Image Processing, 2007, Vol.16, No.3, pp.741-758.

[11] Tsai, C. M., and Yeh, Z. M., "Contrast enhancement by automatic and parameter-free piecewise linear transformation for color images", IEEE Transactions on Consumer Electronics, 2008, Vol.54, No.2, pp.213-219.

[12] Kim, J. Y., Kim, L. S., \& Hwang, S. H., “An advanced contrast enhancement using partially overlapped subblock histogram equalization", IEEE Transactions on Circuits and Systems for Video Technology, 2001, Vol. 11 , No. 4, pp, 475-484

[13] Marsi, S., Impoco, G., Ukovich, A., Ramponi, G., and Carrato, S., "Using a recursive rational filter to enhance color images", IEEE Transactions on Instrumentation and Measurement, 2008, Vol.57, No.6, pp.1230-1236.

[14] Ford, A., and Roberts, A.1998. Colour space conversions. Westminster University, London.

[15] Starck, J. L., Fadili, J., \& Murtagh, F, "The undecimated wavelet decomposition and its reconstruction", IEEE Transactions on Image Processing, 2007, Vol. 16, No. 2, pp. 297-309.

[16] MICT image quality evaluation database: http://mict.eng.u-toyama.ac.jp/mictdb.html. 